A PRAGMATIC TRIAL IN THE RIO DE JANEIRO SUBWAY TO CAPTURE SMOKERS FOR A OUITLINE: METHODOLOGICAL CHALLENGES AND OPPORTUNITIES

doi:10.1136/jech.2011.142976f.41

${ }^{1}$ A Szklo, ${ }^{*}$ E Coutinho, ${ }^{3} \mathrm{M}$ Reichenheim. ${ }^{1}$ Brazilian National Cancer Institute, Rio de Janeiro, RJ, Brazil; ${ }^{2}$ National School of Public Health, Rio de Janeiro, RJ, Brazil; ${ }^{3}$ Institute of Social Medicine, Rio de Janeiro, RJ, Brazil

Objective According to WHO, smoking is an important cause of death in many countries. To encourage smoking cessation, persuasive messages can be used to raise smokers' risk perception. This work discusses challenges and solutions in designing a study to evaluate the impact of two different communication strategies ("gains from quitting" vs "losses from continuing smoking") in encouraging calls to a Quitline.

Methodology A pragmatic intervention study was conducted in two subway stations for 4 weeks. Large posters containing non-age specific images and texts, based on the theme "shortness of breath", were displayed on central dividing columns on the boarding platforms. Call rates from the selected stations, and respective rate ratios, overall and per study week, were calculated.

Results Passengers who were smokers, exposed to the positivecontent message, called on average 1.7 times more often than those exposed to the negative-content message $(p=0.01)$. Moreover, call rate ratios did not decline over the 4 weeks of the study (multiplicative interaction $\mathrm{p}=0.40$ ).

Conclusions The effectiveness findings suggest that anti-smoking campaigns could use positive-content messages in order to recruit a larger smoker population. The proposed methodology can also be used to evaluate effectiveness of messages for "capturing' individuals with other health problems (eg, alcohol abuse), thereby increasing its potential impact.

\section{P1-350 A SNAPSHOT OF THE STRIKING DECREASE IN CIGARETTE SMOKING PREVALENCE IN BRAZIL BETWEEN 1989 AND 2008}

doi:10.1136/jech.2011.142976f.42

${ }^{1}$ A Szklo, ${ }^{*}$ L Almeida, ${ }^{1} \mathrm{M}$ Sampaio, ${ }^{3} \mathrm{D}$ Malta, ${ }^{4} \mathrm{~S}$ Mirza, ${ }^{2} \mathrm{R}$ Caixeta, ${ }^{5} \mathrm{~V}$ Figueiredo, ${ }^{1} \mathrm{M}$ Szklo. ${ }^{1}$ Brazilian National Cancer Institute, Rio de Janeiro, Brazil; ${ }^{2}$ Health Surveillance Agency, Brasilia, Distrito Federal, Brazil; ${ }^{3}$ Center for Disease Control, Atlanta, Georgia, USA; ${ }^{4}$ Panamerican Health Organization, Washington, DC, USA; ${ }^{5}$ Federal University of Rio de Janeiro, Rio de Janeiro, Brazil

Objective To evaluate the differences in cigarette smoking prevalence in Brazil between 1989 and 2008.

Methodology We compared absolute and relative differences in smoking prevalence, overall and stratified by selected socio-demographic variables and birth cohort (20-year interval from 1925 to 1934 onwards). Data were obtained from National Household Survey on Health and Nutrition (1989, n=39969) and Global Adult Tobacco Survey (2008, $n=38461$ ). Generalised linear models with binomial family distribution, and either gaussian or logarithmic link function, were specified in order to obtain estimates, as well as to assess potential effect modification.

Results Crude and adjusted overall differences in smoking prevalences between 1989 and 2008 were, respectively: absolute, 15.4\% and 11.8 ; relative, $47.5 \%$ and $38.7 \%$. We observed the highest declines in smoking prevalences among individuals aged 25-34 years-old (additive or multiplicative interaction $\mathrm{p}_{\mathrm{s}}<0.001$ ) and those with 8 years of schooling or more (multiplicative interaction $\mathrm{p}<0.001$ ). Moreover, while stratifying by birth cohort, we found that, only in the absolute scale and with the exception of the youngest birth cohort (ie,1965-1974), men presented higher reductions than women (additive interaction terms $<0.001$ ).

Conclusions A large amount of laws against tobacco consumption have been adopted in Brazil since 1986, which may have contributed to the observed decline in smoking prevalence. It is of paramount importance to better understand the effectiveness of tobacco control actions implemented in a country and the evolution of its tobacco epidemic in order to improve/develop actions targeted to those who continued to smoke and/or started smoking in a "more hostile" environment.

\section{P1-351 EFFECT OF COFFEE CONSUMPTION ON ALL-CAUSE AND TOTAL CANCER MORTALITY: FINDINGS FROM THE JACC STUDY}

doi:10.1136/jech.2011.142976f.43

${ }^{1} \mathrm{~A}$ Tamakoshi, ${ }^{1} \mathrm{Y}$ Lin, ${ }^{2} \mathrm{M}$ Kawado, ${ }^{1} \mathrm{~K}$ Yagyu, ${ }^{1} \mathrm{~S}$ Kikuchi, ${ }^{3} \mathrm{H}$ Iso. ${ }^{1}$ Aichi Medical University School of Medicine, Aichi, Japan; ${ }^{2}$ Fujita Health University School of Medicine, Aichi, Japan; ${ }^{3}$ Osaka University Graduate School of Medicine, Osaka, Japan

Coffee consumption is known to be related to various health conditions. Recently, its antioxidant effects have been suggested to be associated with all-cause or cancer mortality by various cohort studies. However, there has been only one small Asian cohort study that has assessed this association. Thus, we tried to assess the association of coffee with all-cause and total cancer mortality by conducting a large-scale cohort study in Japan. A total of 97753 Japanese men and women aged 40-79 years were followed for 16 years from 1988 to 1990 . HRs and 95\% CIs of all-cause and total cancer mortality in relation to coffee consumption were calculated from proportional-hazards regression models. A total of 19532 deaths occurred during the follow-up period; $34.8 \%$ of these deaths were caused by cancer. The all-cause mortality risk decreased with increasing coffee consumption in both men and women, with a risk elevation at the highest coffee consumption level ( $\geq 4$ cups/day) compared with the $2^{\text {nd }}$ highest consumption level in women, although the number of subjects evaluated at this level was small. No association was found between coffee consumption and total cancer mortality among men, whereas a weak inverse association was found among women. The present cohort study among the Japanese population suggested that there are beneficial effects of coffee on all-cause mortality among both men and women. Furthermore, the results showed that coffee consumption might not be associated with an increased risk of total cancer mortality.

\section{P1-352 INEQUALITIES IN HEALTH: RELATIVE, ABSOLUTE, AND AN ETHICAL DIMENSION}

doi:10.1136/jech.2011.142976f.44

\section{A Tannahill, ${ }^{*}$ L Gruer. NHS Health Scotland, Glasgow, UK}

Introduction Discussions on inequalities in health and trends in these often concentrate explicitly or implicitly on relative inequality. This paper explores practical and ethical implications of that tendency.

Methods Trends in relative and absolute inequalities in coronary heart disease in Scotland between 1991 and 2006 were assessed using existing data. A hypothetical alternative scenario, featuring different trends, was devised and its implications compared with those of the actual scenario.

Results The 'headline' actual trend was an increase in relative inequality between the most and least deprived population groups. However, there was also a reduction in absolute inequality, and both groups benefited from substantially reduced mortality rates. In the hypothetical scenario relative inequality would have lessened, but at 